\title{
Wavefront aberration reconstruction from tangential refractive powers measured with spatial dynamic skiascopy
}

\author{
Sergio Barbero \\ Instituto de Óptica, Consejo Superior de Investigaciones Científicas (CSIC), Serrano 121, Madrid 28006, \\ Spain (sergio.barbero@csic.es)
}

Received 14 September 2012; revised 16 November 2012; accepted 17 November 2012; posted 19 November 2012 (Doc. ID 176288); published 14 December 2012

\begin{abstract}
The aim of this work was to study, using numerical simulations, the attainable level of accuracy to reconstruct the wavefront aberrations from tangential refractive power data measured with dynamic skiascopy. Two mathematical methods have been implemented. The first one is based on curve integration of the curvature data, previously interpolated with cubic splines. The second one reconstructs the three-dimensional wavefront surface, represented by a Zernike polynomial expansion, using a two-step least-squares method. The different factors affecting the reconstruction-noise, sampling, and wavefront patterns-were quantified. The results provide useful information to design more efficient experimental setups based on spatial dynamic skiascopy. (C) 2012 Optical Society of America
\end{abstract}

OCIS codes: $\quad 330.4460,080.1010,330.7327$.

\section{Introduction}

Retinoscopy, also called skiascopy, is a procedure to measure refractive errors using light reflected back from the retina (fundus reflex). Particularly, this study deals with an automatized type of skiascopy, which measures the time that the fundus reflex takes to move from one pupil position to another. From this time measurement, the speed of motion is obtained. Finally, the refractive power is directly proportional to the speed of motion (see Chap. 18 in [1]).

An extension of this technology, spatial dynamic skiascopy, was proposed for wavefront aberrometry by the Nidek Corporation: the so-called optical path difference (OPD)-Scan system, now in its third version (OPD-Scan III). The ideas behind of the OPD-Scan were described in a patent by Fujieda [2], and subsequently summarized in a paper [3]. An infrared slit beam, in the tangential plane, is projected onto the retina, and subsequently the light reflected back from the retina is captured by an array

$1559-128 \mathrm{X} / 12 / 368599-07 \$ 15.00 / 0$

(C) 2012 Optical Society of America of photodetectors conjugated with the emmetropic retina. A chopper wheel rotates the slit beam plane of incidence. The photodetectors measure time differences at different locations within a reference plane. These time differences can be converted to refractive power errors, thus obtaining a refractive power map.

A relevant question is whether, from this refractive power map, there is a method capable of reconstructing the wave aberration function with enough accuracy. It must be noted that a first and unavoidable limitation of such a method is that tip/tilt components of the wavefront cannot be retrieved from the power data because the curvature (power) is always zero for these components. However, this is not so critical because these terms are usually ignored in wavefront aberration reconstruction.

One other inherent limitation is due to what has been called the skew ray issue [4]. Only the rays of light reflected back from the retina that are included in the scanning plane (tangential plane) are collected by the photodetectors. As a consequence, only the tangential component of the power (tangential curvature of the wavefront) is measured. This limitation has been also considered by Fujieda and Yukinobu [5]. To avoid this 
problem, Fujieda and Yukinobu proposed a new system [5] where not only the tangential but also the sagittal (orthogonal direction to the tangential) refractive power was measured. However, such an experimental system is much more complicated, and, to our knowledge, it has not been yet implemented in a commercial device. Therefore, the mathematical problem consists of trying to reconstruct a surface (wavefront aberration) from the tangential curvature data (tangential refractive power) of that surface. Hieda and Kinoshita [6] published a method to solve this optical problem, which hereafter will be referred as Hieda's algorithm.

Hieda's algorithm recovers the wavefront surface through curve reconstruction by means of direct integration of the curvature data along the different meridians. However, as reported by MacRae and Fujieda [3], the OPD-Scan system measures curvature only at diameters of 2.0, 3.2, 4.4, and $5.5 \mathrm{~mm}$. Direct integration of such sparse sampling is not an appropriate procedure. Also, Hieda's algorithm is based on the assumption that the wavefront tangential curvature at a specific point is proportional to the radial first derivative of the wavefront divided by the radial coordinate at that point (first equation in [6] ). However, this approximation is only accurate when the angle of intersection of the normal to the wavefront and the optical axis is small enough to be almost equal to the wavefront radial slope (see [7]). Therefore, this assumption could lead to significant reconstruction errors for highly aberrated wavefronts. Moreover, it is an unnecessary assumption, as will be shown.

In this article, two novel methods have been derived for reconstructing the wavefront aberration function from tangential curvature data as measured with dynamic skiascopy. The potential accuracy of these algorithms has been quantified by means of numerical simulations.

\section{Methods}

Two different reconstruction algorithms have been implemented. The first one is a variation of Hieda's algorithm, also based on curve integration of the curvature data, but including important changes. The second algorithm reconstructs the wavefront surface, represented by a Zernike polynomial expansion, by a two-step least-squares method.

\section{A. Curve Integration Reconstruction}

Given the curvature of a plane curve, an important theorem in differential geometry establishes that there exists only one curve expressed as a function of the vector position having that curvature function [8]. Therefore, it is plausible to propose an algorithm based on reconstructing the plane curves embedded in the wave aberration surface. These curves, generated by intersecting the tangential planes with the wave aberration surface, can be explicitly described in Cartesian coordinates as $y(x)$, where $x$ is the distance from the surface vertex. The equation to be solved is the one relating the curvature of $y$ with its first $\left(y^{\prime}\right)$ and second derivatives $\left(y^{\prime \prime}\right)$ with respect to $x$ :

$$
y^{\prime \prime}=K_{t}\left(1+y^{\prime 2}\right)^{3 / 2},
$$

where $K_{t}$ is the curvature of the curve.

Equation (1) is an ordinary differential equation of second order. If initial values for the first and second derivatives are set, this equation can be solved analytically $[9,10]$ for $y^{\prime}$ using an auxiliary function $\psi$ (see [10]). The $\psi$ function is the integrated curvature,

$$
\psi(x)=\int_{0}^{x} K_{t}(\xi) \mathrm{d} \xi
$$

and $y^{\prime}$ is obtained with equation

$$
y^{\prime}(x)=\frac{\psi(x)+\alpha}{\sqrt{1-(\psi(x)+\alpha)^{2}}},
$$

where the constant $\alpha$ is given by

$$
\alpha=\frac{y^{\prime}(0)}{\sqrt{1+\left(y^{\prime}(0)\right)^{2}}} .
$$

Finally $y$ is obtained by direct integration of $y^{\prime}$.

The OPD-Scan instrument reference coordinate system is relative to the apex of the cornea [3]. It is reasonable to assume that, at this point, the slope (first derivative) of the curve is zero and that the central vertex curvature (power) is measured somehow. As mentioned above, in such a case, an analytical solution could be found if the curvature function were known. However, given that only numerical curvature data are measured, a numerical procedure is needed. If the sampling data in the radial coordinate are not densely and uniformly distributed, direct integration to obtain the auxiliary function $\psi$ [10] yields significant errors. Curve fitting or interpolation is a solution to such a problem. Interpolation with piecewise polynomials is particularly suitable. Specifically, we found that curve interpolation with cubic splines $[11,12]$ offered the best results.

In summary, the algorithm comprises the following steps: (1) cubic spline interpolation of the curvature function for each meridian, (2) solving Eq. (2), numerical integration of the curvature function to obtain the auxiliary function $\psi,(3)$ estimation of the first derivative of the curve using the $\psi$ auxiliary function with the help of Eqs. (3) and (4), and (4) numerical integration (trapezoidal rule) of the curve slope $\left(y^{\prime}\right)$ to obtain the curve points. The three first steps are not present in Hieda's algorithm.

Finally, from the integrated curves embedded in the wavefront surface, the wavefront aberration function was fitted using least squares with a Zernike polynomial expansion.

\section{B. Modal Surface Fitting Reconstruction}

This method applies least-squares fitting of the surface with Zernike polynomials. This is a widely used technique in wavefront reconstruction when input data are transverse ray aberrations, for instance in 
Hartmann-Shack sensors (see, for instance [13] and its references). This generic method has been adapted to our specific problem with some relevant modifications.

The wavefront aberration function in polar coordinates $u(r, \theta)$ can be represented as a Zernike polynomial expansion: $u(r, \theta)=\sum_{k=1}^{n} C_{k} Z_{k}(r, \theta)$. The second derivative with respect to the radial coordinate $\left(u_{r r}(r, \theta)\right)$ gives

$$
u_{r r}(r, \theta)=\sum_{k=1}^{n} C_{k} \frac{\partial^{2} Z_{k}(r, \theta)}{\partial r^{2}}
$$

If the angular coordinate $(\theta)$ is set as a fixed parameter (tangential plane), the two-dimensional function $u_{r r}(r, \theta)$ is transformed to a one-dimensional family of functions. Therefore, it is possible to apply Eq. (1) to these one-dimensional functions in order to obtain a family of equations for the tangential curvature at each tangential plane:

$$
u_{r r}(r, \theta)=K_{t}(r, \theta)\left(1+u_{r}(r, \theta)^{2}\right)^{3 / 2},
$$

where $u_{r}(r, \theta)$ is the first radial derivative and $K_{t}(r, \theta)$ is the tangential curvature function (input data). Now, in a first step of the algorithm, the second radial derivate is approximated with the tangential curvature, $K_{t}(r, \theta)\left(1+u_{r}(r, \theta)^{2}\right)^{3 / 2} \sim K_{t}(r, \theta)$,

$$
K_{t}(r, \theta)=\sum_{k=1}^{n} C_{k} \frac{\partial^{2} Z(r, \theta)}{\partial r^{2}}
$$

The problem is reduced to solve a system of linear equations [Eq. (7)]. It should be noted that the polynomial expansion using the second derivatives of Zernike polynomials is not orthogonal. Several procedures to recover an orthogonal expansion have been proposed $[7,14]$. However, the lack of orthogonality of the second derivative functions is in practice, as mentioned elsewhere [15], not so problematic when the system of linear equations is highly overdetermined (larger number of data with respect to the number of base functions), as it is in this case. Particularly, we used a sixth-order Zernike polynomial expansion $(n=29)$, and, as will be shown in the next section, the number of points where the radial curvature is measured takes values typically larger than 1000. Hence, the aforementioned condition is fully justified.

Equation (7) is solved in the least-squares sense using the pseudoinverse, singular value decomposition, with a function implemented in MATLAB (pinv function). Then, a first estimate of the Zernike aberration coefficients is already obtained. In a second step of the algorithm, we go back to the original Eq. (6). This represents a system of nonlinear equations, which cannot be solved using linear algebra techniques.
Therefore, we built a merit function as follows:

$$
\mathrm{MF}=\sum_{i}\left(K_{i}-\tilde{K}_{i}\right)^{2} .
$$

$K_{i}$ and $\tilde{K}_{i}$ are the measured and estimated tangential curvatures, respectively. An iterative optimization procedure is used to minimize the merit function of Eq. (8). At each step of the iteration, the Zernike coefficients of the previous step are modified aiming at decreasing the value of Eq. (8). Applying standard formulas of differential geometry [8], $\tilde{K}_{i}$ are computed analytically from the wavefront represented as a Zernike polynomial expansion. The convergence of the optimization algorithm is highly dependent on the initial guess for the Zernike coefficients. Fortunately, the good initial guess was provided by the solution of Eq. (7). For the optimization, a Levenberg-Marquardt search algorithm, as implemented in the lsqnonlin MATLAB function, was used.

\section{Numerical Tests}

In order to evaluate and quantify the potential accuracy of the two proposed algorithms, several numerical simulations were performed. Besides the robustness of the algorithm itself, the accuracy depends also on three factors: (1) the shape of the wavefront aberration, (2) the sampling pattern of the experimental curvature data, and (3) the experimental errors in the curvature data.

In the numerical simulations, the normalized rootmean-square error (NRMSE) was used as a measure of the differences between the nominal values of the Zernike aberrations and the reconstructed values. The NRMSE is the root-mean-square error divided by the range of observed values. It is expressed as a percentage.

\section{Wave Aberration Function Tests}

Campbell proposed [16] three specific artificial test eyes to be used as a tool to evaluate the feasibility of different types of devices to measure ocular aberrations. For comparative purposes, the wave aberrations corresponding to these test eyes were used in the numerical simulations. All the information of these tests is provided in reference [16]. Figure 1 shows the wavefront aberration patterns. Nominally, one of the test eyes (A4) has a rotationally symmetric wavefront (only defocus and spherical aberration), while the other two (A3 and L2) show significant amounts of asymmetrical aberrations. The curvatures of the wavefront patterns (input data for the simulations) were obtained using standard formulas of differential geometry [8].

\section{Sampling Data}

In the OPD-Scan system, the whole eye is scanned with a rotational slit beam, along different tangential planes, in $1 \mathrm{deg}$ steps. For each tangential plane power data are provided in four radial positions from 


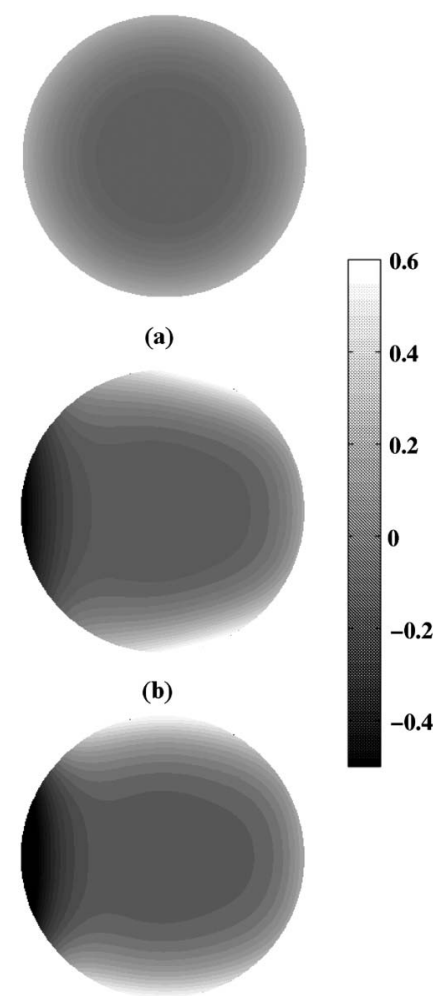

(c)

Fig. 1. Wavefront aberration patterns used in the simulations (Campbell's eye tests [16]): (a) test eye A4, (b) test eye A3, and (c) test eye L2. The grayscale pattern represents wave aberration heights in micrometers.

the reference center [3]: $1.15,1.65,2.2$, and $2.75 \mathrm{~mm}$. Hence, the refractive power is measured at 1440 points. This sampling pattern will be referred to as S1. A new version of the system (OPD-Scan III) extends the number of rings (up to seven zones and 4.75 of maximum radial coordinate), but apparently it does not increase the density within the four inner zones.

As already mentioned, the OPD-Scan sampling is very sparse in the radial coordinate. In order to evaluate the benefits of a experimental device with a denser number of radial photodetectors, a sampling pattern suggested by Atchison [4] was also evaluated, where the measured points are equally spaced in the radial coordinate each $0.3 \mathrm{~mm}$. This sampling has 3240 measuring points. This will be referred to as S2. In addition, a sampling pattern with the same radial density as that of Atchison's pattern, but with a more sparse angular density, was also evaluated in order to have the same number of points (1440) as the OPD-Scan system. This pattern will be denoted as $\mathrm{S} 3$.

\section{Noise Model}

Unfortunately, there are no available data in the literature on the accuracy of the measurement of local refractive powers using dynamic skiascopy. Because of this, different levels of additive Gaussian noise were added to the curvature data in the simulations.
The error is quantified with the signal-to-noise ratio (SNR), using decibel units: $\mathrm{SNR}=10 \log _{10}(K / N)$, where $K$ is the signal and $N$ is the noise. SNRs ranging from $20 \mathrm{~dB}$ (high noise) to $50 \mathrm{~dB}$ (low levels) were simulated. In addition, the reconstruction without the addition of noise (zero noise) was also evaluated.

\section{Results}

Table 1 gives the NRMSE (\%) obtained with the reconstruction algorithms for the different tests and sampling patterns. No noise was added to the curvature data at this stage.

For all the zero-noise cases, the accuracy in the reconstruction was very high (below $0.1 \%$ ). Also, the surface fitting algorithm provided better results than the curve integration algorithm, except in the case of the eye test A4 (rotationally symmetric). For the surface fitting reconstruction, there is a significant improvement when using sampling patterns with a higher density in the radial coordinate (S2, S3 compared to pattern $\mathrm{S} 1$ ), whereas this improvement is not obtained when using the curve integration algorithm. The rotationally symmetric wavefront is recovered substantially better than the nonsymmetrical wavefronts.

Figure 2-4 show the NRMSE (\%) in the reconstruction algorithms for different levels of noise and for the eye tests A4, A3, and L2, respectively. The Figs. 2(a) and 2(b)-4(a) and 4(b) show the NRMSEs for the surface fitting and curve integration reconstruction algorithms, respectively.

By direct comparison to the zero-noise results, it is obvious that the presence of noise significantly affects the accuracy of the reconstruction of both algorithms. Even with very low levels of noise (50 dB), the NRMSE increases up to $2 \%-30 \%$. The differences in the accuracy of the reconstruction with both algorithms depend on the level of noise. For example, for the surface fitting reconstruction, the NRMSE (test eye A3 and sampling pattern S1) is reduced from $22.3 \%$ with high noise $(20 \mathrm{~dB})$ to $14.9 \%$ with low noise $(50 \mathrm{~dB})$. As for the zero-noise case, the rotationally symmetric wavefront is recovered significantly better than the nonsymmetrical wavefronts; in average across sampling patterns and noise levels, the difference is in percentage by $121.47 \%$.

Contrary to the results for the zero-noise case, the presence of noise strongly reveals the importance of increasing the number of samples in the radial coordinate. The NRMSE is reduced in percentage by $57.97 \%$ (average across test eyes, and noise levels) when S2 is used instead of S1. The differences are much smaller between S2 and S3 (18.99\% average across test eyes and noise levels). This result shows that the density of sampling points along the radial coordinate is much more critical than the density along the angular coordinate in the reconstruction performance.

The accuracy in the reconstruction using the surface fitting method is $55.58 \%$ (average for all the 
Table 1. NRMSE (\%) of the Algorithms for Different Tests (A4, A3, and L2) and Sampling Patterns (S1, S2, and S3)

\begin{tabular}{|c|c|c|c|c|c|c|}
\hline & \multicolumn{3}{|c|}{ Curve Integration Reconstruction } & \multicolumn{3}{|c|}{ Surface Fitting Reconstruction } \\
\hline & A4 & A3 & L2 & A4 & A3 & L2 \\
\hline $\mathbf{S 1}$ & $1.1965 \mathrm{E}-04$ & $1.0858 \mathrm{E}-01$ & $1.0570 \mathrm{E}-01$ & $3.2686 \mathrm{E}-04$ & $4.8926 \mathrm{E}-03$ & $3.1762 \mathrm{E}-03$ \\
\hline S3 & $1.2304 \mathrm{E}-04$ & $1.5709 \mathrm{E}-01$ & $1.4579 \mathrm{E}-01$ & $7.1466 \mathrm{E}-05$ & $9.1738 \mathrm{E}-04$ & $7.4193 \mathrm{E}-04$ \\
\hline
\end{tabular}

${ }^{a}$ No additive noise.

noise levels, sampling patterns and test eyes) better than the reconstruction using the curve integration method.

Finally, Fig. 5 compares the results of the numerical reconstructions with that of the experimental reconstructions measured by Campbell [16]. For this comparison, the numerically reconstructed wavefronts were expressed as a Zernike polynomial expansion. This figure shows, for a high noise level $(20 \mathrm{~dB})$, the absolute errors in the Zernike coefficients (difference with respect to the nominal values) of Campbell's measurements and our own results.

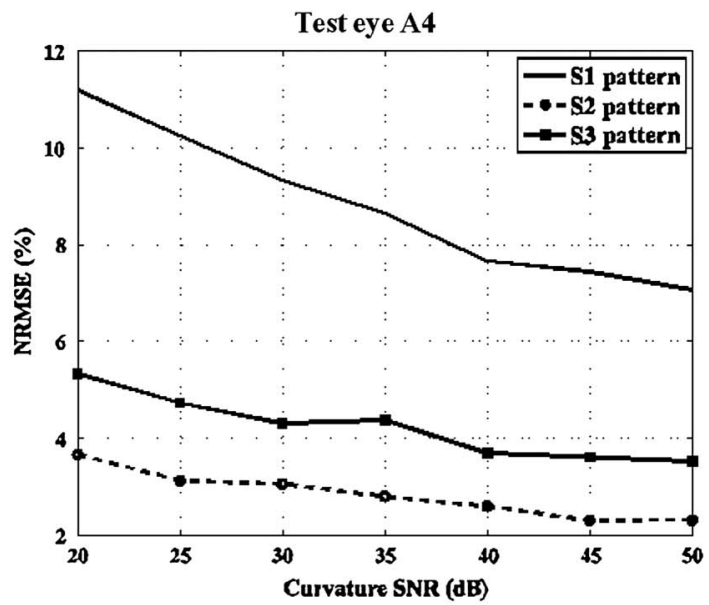

(a)

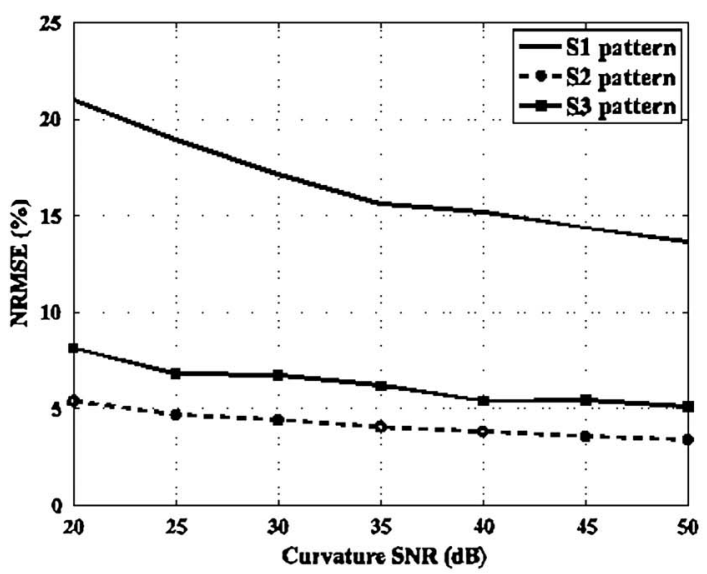

(b)

Fig. 2. NRMSE, expressed as a percentage, as a function of curvature SNR in decibels for test eye A4. Sampling patterns S1, S2, and S3 are plotted with a solid curve, a dashed curve with circles, and a solid curve with squares, respectively. Numerical reconstruction for (a) the modal fitting and (b) the curve integration reconstruction algorithms.

\section{Discussion}

The goal of this study was to propose two novel methods to reconstruct wavefront aberrations from tangential curvature data and to study the effects of different factors-noise, sampling, and wavefront patterns-in the reconstruction accuracy.

Several works [17-20] have compared the aberrations reconstructed by means of dynamic skiascopy with other types of wavefront sensors, showing different results. Nevertheless, such comparisons do not provide a measurement of the reconstruction accuracy because none of the aberrometers is free of its

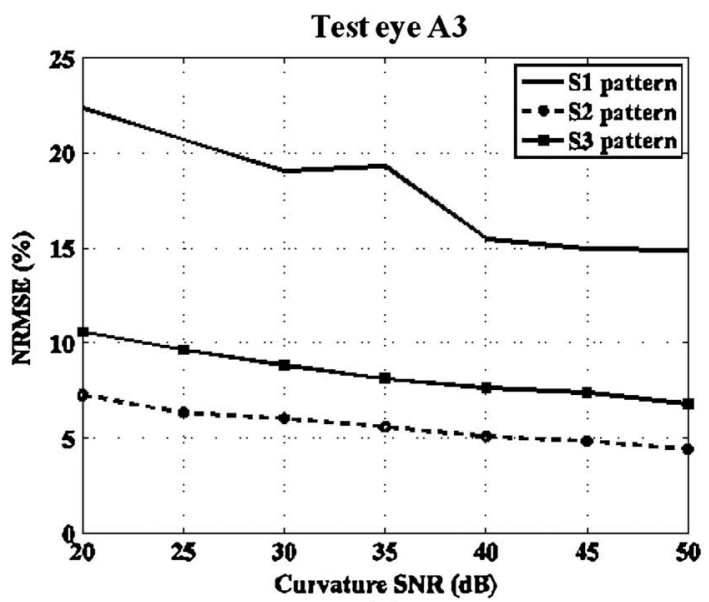

(a)

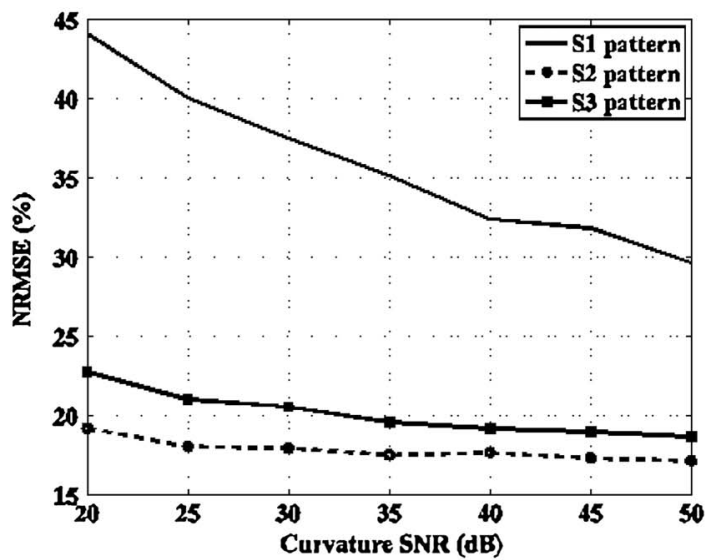

(b)

Fig. 3. NRMSE, expressed as a percentage, as a function of curvature SNR in decibels for test eye A3. Sampling patterns S1, S2, and S3 are plotted with a solid curve, a dashed curve with circles, and a solid curve with squares, respectively. Numerical reconstruction for (a) the modal fitting and (b) the curve integration reconstruction algorithms. 


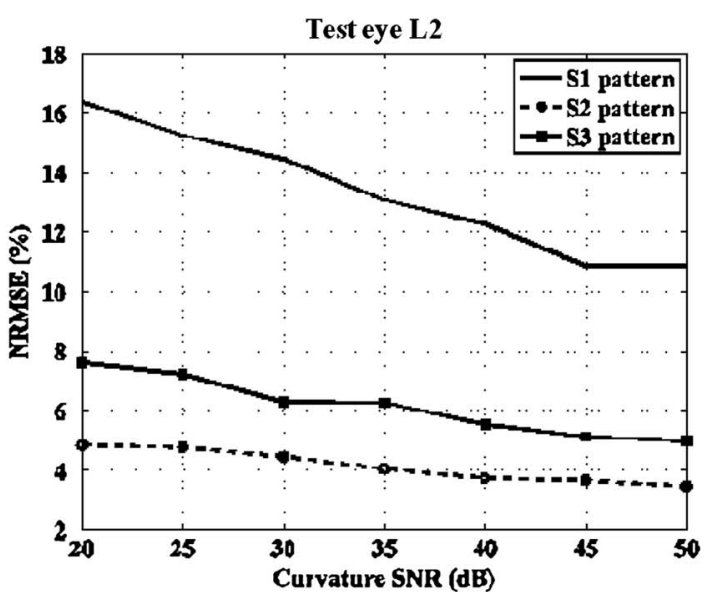

(a)

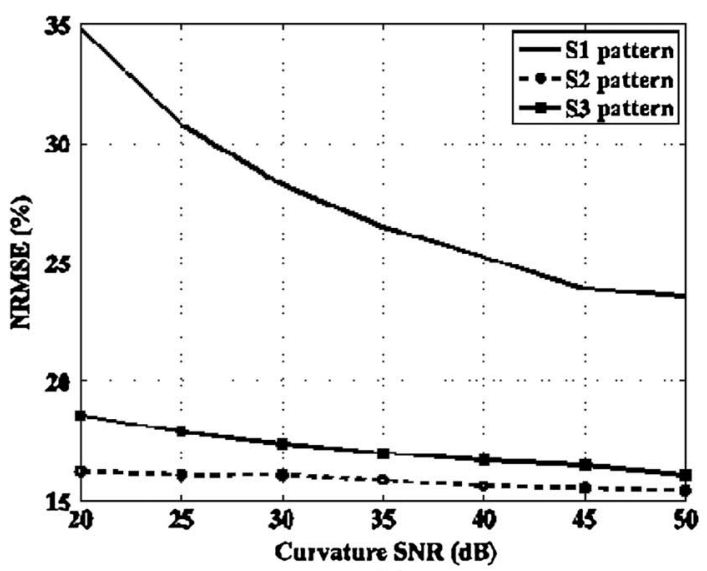

(b)

Fig. 4. NRMSE, expressed as a percentage, as a function of curvature SNR in decibels for test eye L2. Sampling patterns S1, S2, and S3 are plotted with a solid curve, a dashed curve with circles, and a solid curve with squares, respectively. Numerical reconstruction for (a) the modal fitting and (b) the curve integration reconstruction algorithms.

own reconstruction errors. However, these studies supplied valuable information in the repeatability error estimation for different aberration coefficients. Burakgazi et al. [18] obtained repeatability errors ranging from 0.03 to $0.12 \mu \mathrm{m}$ and Zadok et al. [21] from 0.03 to $0.07 \mu \mathrm{m}$. Cerviño et al. [19] did not find a good agreement between the aberrations measured with an OPD-Scan with respect to those measured by a Hartmann-Shack aberrometer. Hieda and Kinoshita [6] also found some significant differences for large pupils compared with another commercial Hartmann-Shack aberrometer (Zywave). Overall, repeatability errors are also relevant and are likely to be affected by the systematic errors studied in this work.

Regarding accuracy, to our knowledge only two studies have studied it experimentally. Hieda and Kinoshita [6] used three artificial eyes with different levels of second-order aberrations, although higherorder aberrations were not considered. The most extensive work is that of Campbell [16], who built three artificial eyes, with known aberrations, to test the

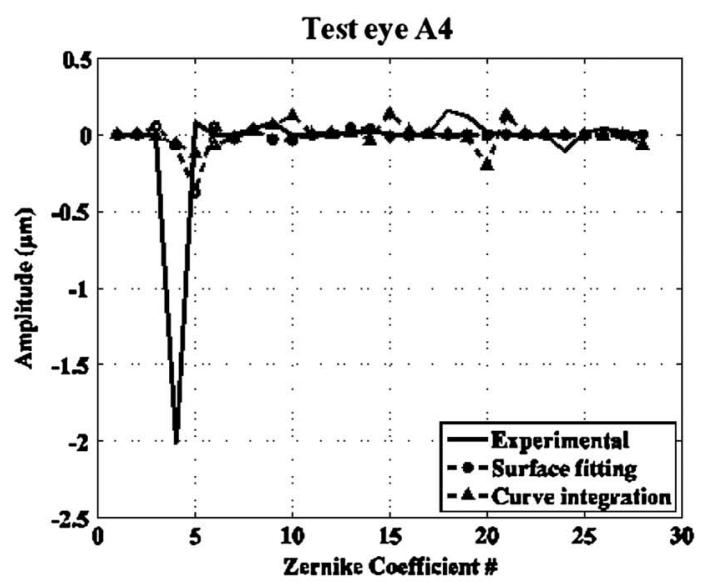

(a)

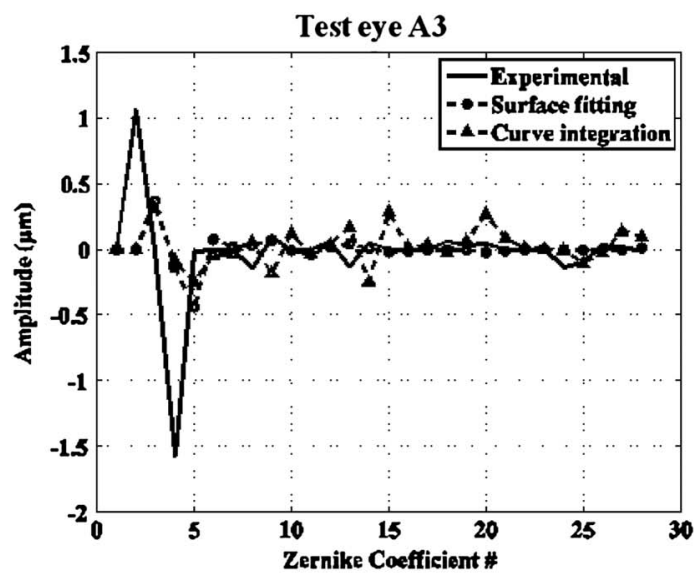

(b)

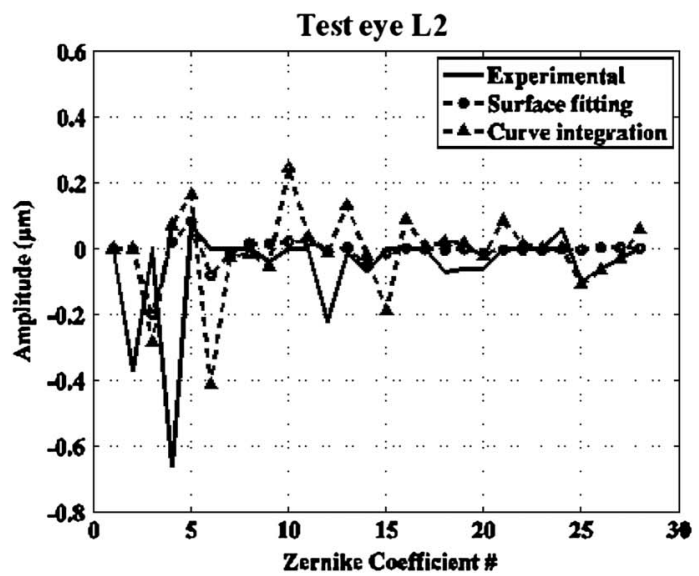

(c)

Fig. 5. Errors in the reconstruction of the Zernike coefficients of the reconstructed wavefronts, both experimentally (as reported in [16]) and numerically. Curvature SNR was $20 \mathrm{~dB}$. Sampling pattern was S1. Test eyes: (a) A4, (b) A3, and (c) L2. Experimental data, surface fitting, and curve integration reconstructions are plotted with a solid curve, a dashed curve with circles, and a dashed curve with triangles, respectively.

feasibility of wavefront sensors to measure aberrations. He included the OPD-Scan aberrometer in his study and found significant differences in the aberrations measured with this device compared to 
the nominal reference values. These differences are particularly relevant for low-order Zernike aberrations (as shown in Fig. 5). Compared to the results from our simulations, Campbell's errors are higher than those obtained using the surface fitting method but not clearly with respect to the reconstruction using the curve integration method (Fig. 5). It should be noted that, for this comparison, we added a highlevel noise $(20 \mathrm{~dB})$ to the curvature data. We should also note that, in Campbell's experiments, all the sources of errors are mixed together, whereas our theoretical method allows separating the contribution to the reconstruction error of the different factors, which we have analyzed in this study.

The results shown in this paper stress the role, in the final performance of the reconstructions algorithms, of the presence of noise in the experimental data. This is probably because tangential curvatures only describe partially the wavefront surface; in other words, the same tangential curvature data could be derived from different wavefronts. Therefore, when adding noise to these data, the number of wavefronts that could be associated to them can rapidly increase, making the search for the real solution more difficult.

The shape of the wavefront to be reconstructed affects the accuracy of the algorithms in two different ways. The curve integration involves two numerical integrations: one using curvatures, and the other using slope data. The error made in these integrations increases when these magnitudes change very fast, which occurs for highly nonspherical (nonconstant curvature) wavefronts. The surface fitting reconstruction makes a first estimation of the reconstructed wavefront approximating the second radial derivative with the tangential curvature. Therefore, wavefronts in which this approximation is not appropriate are more sensitive to this error. Besides these effects, both algorithms implicitly rely on the assumption that the tangential curvatures dominate over the sagittal ones. This is particularly so for the surface fitting reconstruction algorithm, where the surface is globally reconstructed ignoring the sagittal component of the curvature data. As a consequence, nonrotationally symmetric wavefronts are less accurately reconstructed, as clearly shown by our results in Table 1 .

The results of this work provide valuable information for future design of new experimental setups in dynamic skiascopy. For instance, this study shows that increasing the radial density of sampling points is a critical issue (something already suspected by Hieda and Kinoshita [6]) to improve the accuracy of the reconstruction algorithm, although, obviously, such a system is experimentally more complicated to implement.

Finally, it should be noted that refractive power (also called vergence) maps can be directly applied in the clinical practice [7]. Hence, it could be argued that, in many cases, it is not necessary to reconstruct the wavefront aberration from these maps. However, there are several applications where wave aberration reconstruction is very useful, for instance, when the simulation of visual performance is required. Finally, we must not forget that slit-based skiascopy setups provide only tangential, not total, refractive power maps.

I would like to thank Lourdes Llorente for valuable discussions and for encouraging me to carry out this work. I also would like to thank Mar Zugaldia for her motivation and her contribution at the early stages of this work. The manuscript has been improved thanks to the comments of the anonymous reviewers.

\section{References}

1. W. J. Benjamin and I. M. Borish, Borish's Clinical Refraction (Butterworth Heinemann/Elsevier, 2006).

2. M. Fujieda, "Ophthalmic measurement apparatus having plural pairs of photoreceiving elements," U.S. patent 5,907,388 (25 May 1999).

3. S. MacRae and M. Fujieda, "Slit skiascopic-guided ablation using the Nidek laser," J. Refract. Surg. 16, S576-S580 (2000).

4. D. A. Atchison, "The skew ray issue in ocular aberration measurement," Optom. Vis. Sci. 83, 396-398 (2006).

5. M. Fujieda and B. Yukinobu, "Ophthalmic measurement apparatus," U.S. patent 7,296,896 (20 November 2007).

6. O. Hieda and S. Kinoshita, "Measuring of ocular wavefront aberration in large pupils using OPD-scan," Semin. Ophthalmol. 18, 35-40 (2003).

7. J. Nam, L. N. Thibos, and D. R. Iskander, "Zernike radial slope polynomials for wavefront reconstruction and refraction," J. Opt. Soc. Am. A 26, 1035-1048 (2009).

8. J. J. Stoker, Differential Geometry (Wiley-Interscience, 1969).

9. A. D. Polianin and V. F. Zaitsev, Handbook of Exact Solutions for Ordinary Differential Equations (CRC, 1995).

10. C. Elster, J. Gerhardt, P. Thomsen-Schmidt, M. Schulz, and I. Weingartner, "Reconstructing surface profiles from curvature measurements," Optik 113, 154-158 (2002).

11. C. Elster, "High-accuracy reconstruction of a function $f(x)$ when only $d / d x f(x)$ or $d 2 / d x 2 f(x)$ is known at discrete measurement points," Proc. SPIE 4782, 12-20 (2002).

12. P. Lancaster and K. Salkauskas, Curve and Surface Fitting: an Introduction (Academic, 1986).

13. G.-M. Dai, "Modal wave-front reconstruction with Zernike polynomials and Karhunen-Loève functions," J. Opt. Soc. Am. A 13, 1218-1225 (1996)

14. E. Acosta, S. Bara, and S. Rios, "Modal projectors for linear operators in Optics," Opt. Commun. 162, 211-214 (1999).

15. J. Nam and J. Rubinstein, "Weighted Zernike expansion with applications to the optical aberration of the human eye," J. Opt. Soc. Am. A 22, 1709-1716 (2005).

16. C. E. Campbell, "A test eye for wavefront eye refractors," J. Refract. Surg. 21, 127-140 (2005).

17. J. J. Rozema, D. E. M. Van Dyck, and M. J. Tassignon, "Clinical comparison of 6 aberrometers. Part 1: technical specifications," J. Cataract Refract. Surg. 31, 1114-1127 (2005).

18. A. Z. Burakgazi, B. Tinio, A. Bababyan, K. K. Niksarli, and P. Asbell, "Higher order aberrations in normal eyes measured with three different aberrometers," J. Refract. Surg. 22, 898-903 (2006).

19. A. Cerviño, S. L. Hosking, and R. Montes-Mico, "Comparison of higher order aberrations measured by NIDEK OPD-Scan dynamic skiascopy and Zeiss WASCA Hartmann-Shack aberrometers," J. Refract. Surg. 24, 790-796 (2008).

20. D. U. Bartsch, K. Bessho, L. Gomez, and W. R. Freeman, "Comparison of laser ray-tracing and skiascopic ocular wavefront-sensing devices," Eye 22, 1384-1390 (2007).

21. D. Zadok, Y. Levy, O. Segal, Y. Barkana, Y. Morad, and I. Avni, "Ocular higher-order aberrations in myopia and skiascopic wavefront repeatability," J. Cataract Refract. Surg. 31, 1128-1132 (2005). 